\title{
Prevalence of Schistosoma mansoni Infection and Associated Risk Factors among School Children in Jiga Town, Northwest-Ethiopia: A Cross-Sectional Study
}

\author{
Ketemaw Wubet ${ }^{1}$ and Destaw Damtie $\mathbb{D}^{2}$ \\ ${ }^{1}$ Finote Selam Teachers College, Finote Selam, Ethiopia \\ ${ }^{2}$ Department of Biology, College of Sciences, Bahir Dar University, Bahir Dar, Ethiopia \\ Correspondence should be addressed to Destaw Damtie; zegades96@gmail.com
}

Received 21 March 2020; Revised 13 June 2020; Accepted 15 June 2020; Published 26 June 2020

Academic Editor: Bernard Marchand

Copyright (c) 2020 Ketemaw Wubet and Destaw Damtie. This is an open access article distributed under the Creative Commons Attribution License, which permits unrestricted use, distribution, and reproduction in any medium, provided the original work is properly cited.

Background. Schistosomiasis, one of the 20 Neglected Tropical Diseases, is a human disease which is caused by infection with Schistosoma spp. In Ethiopia, the intestinal form of schistosomiasis (caused by Schistosoma mansoni) is widely distributed. This study is aimed at assessing the prevalence of $S$. mansoni and associated risk factors among schoolchildren in Jiga town and its surrounding rural "Kebeles", Northwest Ethiopia (Kebele $=$ the smallest administrative unit in Ethiopia). Methods. A crosssectional study was carried out among children from three schools in Jiga town and its surrounding rural "Kebeles". The schools were selected mainly according to their proximity to water bodies that were likely to have been used by the children. A total of 362 randomly selected children were screened for S. mansoni infection. Samples of stool were collected from each pupil and examined by the concentration and microscopic techniques. Semistructured interview questionnaires were administered to the study subjects to identify the possible risk factors of infection with S. mansoni. Data analysis was performed using SPSS version 20 software. Results. The overall prevalence of $S$. mansoni was $15.2 \%$. A higher proportion of $S$. mansoni infection (30.3\%) was observed at Chifarit primary school children followed by Tikur Wuha primary school children (17.3\%) and Jiga 01 primary school children (10.2\%). The habit of contact with river water (AOR, 4.86; 95\% CI, 0.991-23.8; $P=0.051$ ) and the irregular wearing of shoes (AOR, 6.06; 95\% CI, 2.61-14.13; $P=0.001$ ) were risk factors for $S$. mansoni infection. Conclusion. $S$. mansoni infection remains a public health problem in the study area. Snail control, mass deworming, and health promotion are recommended.

\section{Background}

Schistosomiasis, one of the 20 Neglected Tropical Diseases (NTDs) (WHO) [1], is a human disease which is caused by infection with Schistosoma spp. Schistosomes use mammalian definitive hosts and freshwater snail intermediate hosts [2]. Schistosomiasis has been reported from 78 countries. In 2017 , over 220.8 million people required preventive treatment $90 \%$ of whom were from Africa [3]. About $13.6 \%$ of the risk group lives in irrigation schemes. The majority of $S$. mansoni infections occur in Sub-Saharan Africa [4]. In Ethiopia, S. mansoni is widely distributed [5]. About 5.01 million people are thought to suffer from schistosomiasis and 37.5 million are at risk of getting the infection [6].

Globally, schistosomiasis results in 3.3 million disabilityadjusted life years (DALYs). Most infected people live in poor communities without access to safe drinking water and adequate sanitation. An estimated $90 \%$ of infected individuals live in Sub-Saharan Africa, where up to 20 million people also suffer severe chronic health consequences of the disease [7]. In children, schistosomiasis can cause anemia, stunting, and a reduced ability to learn, although the effects are usually reversible with treatment [3]. Elimination of the disease is possible by mass drug administration with 
$40 \mathrm{mg} / \mathrm{kg}$ of praziquantel and integrated control targeting the life cycle [8].

Human infection happens due to exposure to cercariae containing water $[9,10]$. Transmission of Schistosomes relies on contamination of water by excreta, intermediate hosts (snails), and skin exposure to contaminated water [11].

Social, cultural, behavioral, and economic factors interact with local environmental and ecological factors to produce extraordinary variation in the epidemiology of schistosomiasis concerning the prevalence and intensity of infection [2]. Human behaviors such as swimming habits, improper waste disposal, use of river water for different purposes, and wide distribution of intermediate hosts are the major contributing factors for the increased prevalence and wide distribution of schistosomiasis [10].

According to Nute et al. [12], the overall prevalence of $S$. mansoni in the Amhara Region was 6.9\%. Schistosomiasis prevalence in the study area in 1986 was 37.7\% [13]. The presence of Tikur Wuha irrigation dam, irrigation canals to Chifarit and Tikur Wuha "Kebeles", and wetlands in these "Kebeles" are fertile grounds for S. mansoni infection. However, recent studies have not been conducted in the area. Therefore, this study was aimed at investigating the prevalence and associated risk factors of schistosome infection among school children from three primary schools in Jiga town and its surrounding rural "Kebeles", Ethiopia.

\section{Methods}

2.1. Study Design and Period. A cross-sectional study was conducted at the Jiga Health Center from November 2018 to March 2019, among students from three schools (Tikur Wuha, Chifarit, and Jiga 01 primary Schools).

2.2. Study Area. The study was carried out among school children in Jiga town and the surrounding rural "Kebeles", Amhara Region, $372 \mathrm{~km}$ away from Addis Ababa. Jiga town is characterized by; $1850 \mathrm{~m}$ altitude, $1000 \mathrm{~mm}$ mean annual rainfall, and $18-28^{\circ} \mathrm{C}$ temperature range. Three rivers, Tikur Wuha, Giligle Tikur Wuha, and Laza, flow through the study area. Tikur Wuha River is used for bathing, cloth washing, irrigation, recreation, source of tap water, and other domestic activities. According to the Central Statistical Agency (CSA) [14], the town has a total population of 4612 (2217 males and 2395 females). Social service institutions in the town are five private clinics, one major health center, and six schools. A total of 4383 (2160 male, 2223 female) students were enrolled in the three schools in the 2018/2019 academic year.

2.3. Study Population. The study population was students of Tikur Wuha, Chifarit, and Jiga 01 primary Schools who were enrolled in the study period.

2.4. Sampling and Sample Size Determination. The sample size was determined using the following formula [15]:

$$
n=\frac{\mathrm{z} 2 p(1-P) / e 2}{1+(z 2 p(1-p) / e 2 N)}
$$

where $n=$ sample size, $N=$ population size (4383), $z=$ $z$-score (1.96), $e=$ margin of error (0.05), and $p=$ population proportion (37.7\%) [13]. The computed sample size was 334 . Considering some nonresponse rate, $10 \%$ of the samples (33) were added resulting in a total sample size of 367 . Furthermore, an online sample size calculator was employed, and the result was the same [16]. The three schools were selected based on their proximity to a river or bodies of water where snails, suspected to be the vector of schistosomiasis in the area, are present and where children are often seen swimming. Students from these schools were expected as they are more vulnerable to schistosome infection than other schools in the town. Students were selected by stratified random sampling techniques using the school of students as a sampling frame. The number of sample students from each stratum (school) was determined using a proportion allocation formula $n=p^{*} N$, where, $p=$ proportion, $N=$ total sample size. Based on this formula the calculated number of sample students in each school was as follows; Chifarit = $(402 / 4383) 367=34, \quad$ Tikur Wuha $=(1960 / 4383) 367=164$, Jiga $01=(2021 / 4383) 367=169$. The name of all students from grade 1-8 in each school was listed down and arranged alphabetically in separate excel sheets. Then, participant students were selected randomly from each school.

2.5. Sociodemographic Characteristics and Associated Risk Factors. Sociodemographic, behavioral, and risk factor data were collected using a structured interview of the questionnaire. The questionnaire was prepared in English and translated to the Amharic language for simplicity and translated back to English to check the consistency.

2.6. Sample Collection and Processing. About $2 \mathrm{~g}$ of fresh stool specimen was taken from each participant using dry, clean, and prelabeled containers. Stool samples were immediately processed and analyzed. S. mansoni eggs were obtained and identified using the concentration and microscopy methods. The community category of schistosome prevalence was classified as low $(<10 \%)$, medium $(10-50 \%)$, and high $(>50 \%)$ (WHO) [17].

2.7. Data Management and Statistical Analysis. The data collected were encoded and analyzed using SPSS version 20. Descriptive statistics and logistic regression were used to determine the prevalence of $S$. mansoni infection and its association with risk factors. Bivariate and multivariate logistic regression analysis was used to estimate Crude OR and adjusted OR, respectively, at 95\% Confidence Interval (CI). The analysis was made based on age and grade level because of maturity and understanding differences across grade levels and age groups.

2.8. Laboratory Procedure. Both the direct wet mount microscopy and formol-ether concentration (FEC) methods were performed in the Jiga health center laboratory. The test procedures were carried out following standard protocols reported by World Health Organization (WHO) [18]. 


\section{Results}

3.1. Sociodemographic Characteristics. A total of 362 children participated in this study (219 males, 143 females). The mean age of the participants was 12.5 years. The majority $(52.8 \%)$ lay in the grade levels of $5-8$. The residence of study participants showed that $61.9 \%$ of them were urban dwellers. The parents of the majority of the study participants were farmers; 168 (46.4\%) and $118(59.0 \%)$ of the children had poor knowledge about the correct modes of transmission of schistosomiasis (Table 1).

3.2. Behavioral and Environmental Factors for $S$. mansoniInfection. Respondents who used to wear shoes usually, regularly, and sometimes were; $43.1 \%, 33.4 \%$, and $23.5 \%$, respectively. The respective proportions of toilet users and nonusers were $55 \%$ and $45 \%$. Most of the respondents $(58.3 \%)$ reported swimming in rivers and $98.1 \%$ of them had contact with river waters. A significant proportion of the participants (34.8\%) were using river/well waters for drinking (Table 2).

3.3. Prevalence of S. mansoni Infection by Sociodemographic, Environmental, and Behavioral Factors. The overall prevalence of S. mansoni infection was 55 (15.2\%). Based on the bivariable logistic regression, the age groups 7 to 10 (COR, 3.83; 95\% CI, 1.554-9.466; $P=0.004)$ and 11 to 14 (COR, 3.465; CI, 1.449-8.284; $P=0.005$ ), grade levels 1 to 4 (COR, 2.200; 95\% CI, 1.215, 3.984; $P=0.009$ ), and the school (Chifarit) (COR, 3.836; CI, 1.556-9.398, $P=0.003$ ) were risk factors for S. mansoni infection (Table 3). However, in the multivariable logistic regression, the habit of contact with river water (AOR, 4.86; 95\% CI, 0.991-23.8; $P=0.051$ ) and the irregular wearing of shoes (AOR, 6.06; 95\% CI, 2.6114.13; $P=0.001)$ were risk factors for $S$. mansoni infection (Table 3).

\section{Discussion}

The prevalence of S. mansoni infection (15.2\%) was medium [17]. This agreed with the reports from Tanzanian (15.1\%) and Nigerian (17.8\%) [19] and reports in Ethiopia; 20.6\% [10], $15-20 \%$ [20], and 18.9\% [21]. However, it was lower than a report from the same area (37.7\%) [13]. This difference might be due to snail control, chemotherapy, health education, and environmental management and modification [22]. It too may be a result of the country's National Neglected Tropical Disease (NTD) Master Plan which was launched in June 2013. As of September 2015, the Federal Ministry of Health has managed to mobilize support to implement mass drug administration in $100 \%$ of the endemic districts for schistosomiasis [23].

Similarly, the prevalence of S. mansoni infection in the present study was lower than the findings in Tanzania (63.5\%) [19], in Egypt (60\%) [24], in Zimbabwe (50\%), and other findings in Ethiopia such as 37.5\% [2] and 24\% [25]. Schistosome prevalence in the present study was also much lower than $89.9 \%$ [26], 82.8\% [27], 40.1\% [12], and $24.9 \%$ [28]. These variations may be due to variations in diagnosis
TABLE 1: Sociodemographic distribution among school children in Jiga town and its surrounding rural "Kebeles", Amhara Region, Ethiopia, March $2019(n=362)$.

\begin{tabular}{lcc}
\hline Sociodemographic characteristics & Frequency & Percent \\
\hline Sex & & \\
$\quad$ Male & 219 & 60.5 \\
$\quad$ Female & 143 & 39.5 \\
School name & & \\
$\quad$ Chifarit & 33 & 9.1 \\
$\quad$ Tikur Wuha & 162 & 44.8 \\
Jiga 01 & 167 & 46.1 \\
Grade level & & \\
1-4 & 171 & 47.2 \\
5-8 & 191 & 52.8 \\
Age in year & & \\
7-10 & 104 & 28.7 \\
10-14 & 145 & 40.1 \\
$\quad \geq 15$ & 113 & 31.2 \\
Residence & & \\
Jiga town & 224 & 61.9 \\
Rural villages & 138 & 38.1 \\
Parents occupation & & \\
Farmers & 168 & 46.4 \\
Merchants & 130 & 35.9 \\
Gov. employee & 64 & 17.7 \\
Knowledge about schistosomiasis & & \\
$\quad$ Yes & 86.8 \\
$\quad$ No & & \\
\hline
\end{tabular}

TABLE 2: Study participants' responses on associated risk factors among school children in Jiga town and its surrounding rural “Kebeles”, March 2019, $(n=362)$.

\begin{tabular}{lcc}
\hline Associated risk factors & Frequency & Percent \\
\hline Shoes wearing habit & & \\
$\quad$ Sometimes & 156 & 23.5 \\
Usually & 121 & 43.1 \\
$\quad$ Regularly & & 33.4 \\
Use toilet & 199 & \\
$\quad$ Yes & 163 & 55 \\
No & & 45 \\
Swimming in river water & 211 & \\
$\quad$ Yes & 151 & 58.3 \\
$\quad$ No & & 41.7 \\
Contact with river water & 355 & \\
$\quad$ Yes & 7 & 98.1 \\
$\quad$ No & & \\
Drinking water source & 223 & 61.6 \\
Tap & 139 & 34.8 \\
$\quad$ River/well &
\end{tabular}


TABle 3: Prevalence and odds ratios (OR) of S. mansoni infection by sociodemographic, environmental, and behavioral actors of school children in Jiga town and its surrounding rural "Kebeles" (2019).

\begin{tabular}{|c|c|c|c|c|c|c|}
\hline \multirow{2}{*}{ Determinant factor } & \multicolumn{2}{|c|}{ S. mansoni } & \multirow{2}{*}{$P$ value } & \multirow{2}{*}{ COR $(95 \%$ CI $)$} & \multirow{2}{*}{$P$ value } & \multirow{2}{*}{$\operatorname{AOR}(95 \%)$} \\
\hline & Positive & Negative & & & & \\
\hline \multicolumn{7}{|l|}{ Age of participants } \\
\hline $7-10$ & $21(20.2)^{@}$ & $83(79.8)$ & $0.004^{*}$ & $3.831(1.554-9.446)$ & 0.205 & $2.389(0.621,9.19)$ \\
\hline $11-14$ & $27(18.6)$ & $118(81.4)$ & $0.005^{*}$ & $3.465(1.449,8.284)$ & 0.018 & $3.04(1.12,8.21)$ \\
\hline$\geq 15$ & $7(6.2)$ & $106(93.8)$ & & 1 & & 1 \\
\hline \multicolumn{7}{|l|}{ Grade level } \\
\hline $1-4$ & $35(20.5)$ & $136(79.5)$ & $0.009^{*}$ & $2.200(1.215,3.984)$ & 0.371 & $1.849(0.482,7.10)$ \\
\hline $5-8$ & $20(10.5)$ & $171(89.5)$ & & 1 & & 1 \\
\hline \multicolumn{7}{|l|}{ School of participants } \\
\hline Chifarit & $10(30.3)$ & $23(69.7)$ & $0.003^{*}$ & $3.836(1.556,9.398)$ & 0.646 & $1.491(0.272,8.18)$ \\
\hline T/Wuha & $28(17.3)$ & $134(82.7)$ & 0.063 & $1.844(0.966,3.518)$ & 0.513 & $0.629(0.150,2.50)$ \\
\hline Jiga 01 & $17(10.2)$ & $150(89.8)$ & & 1 & & 1 \\
\hline \multicolumn{7}{|c|}{ Knowledge about schistosomiasis } \\
\hline Yes & $8(9.3)$ & $78(90.7)$ & 0.079 & 1 & 0.613 & 1 \\
\hline No & $47(17)$ & $229(83)$ & & $2.036(0.922,4.495)$ & & $0.621(0.149,2.43)$ \\
\hline \multicolumn{7}{|l|}{ Sex of participants } \\
\hline Male & $33(15.1)$ & $186(84.9)$ & 0.935 & $1.025(0.570,1.840)$ & & \\
\hline Female & $22(15.4)$ & $121(84.6)$ & & 1 & & \\
\hline \multicolumn{7}{|l|}{ Residence } \\
\hline Jiga town & $21(15.1)$ & $118(84.9)$ & & 1 & & \\
\hline Rural villages & $34(15.2)$ & $189(84.8)$ & 0.971 & $0.989(0.548,1.786)$ & & \\
\hline \multicolumn{7}{|l|}{ Parents occupation } \\
\hline Farmer & $32(19.1)$ & $136(80.9)$ & 0.157 & $1.882(0.785,4.516)$ & & \\
\hline Merchants & $16(12.2)$ & $115(87.8)$ & 0.824 & $1.113(0.433,2.860)$ & & \\
\hline Gov't employee & $7(11.1)$ & $56(88.6)$ & & 1 & & \\
\hline \multicolumn{7}{|l|}{ River water contact } \\
\hline No & $2(28.6)^{@}$ & $5(71.4)$ & & 1 & & 1 \\
\hline Yes & $53(14.9)$ & $302(85.1)$ & $0.008^{*}$ & $7.948(1.728,36.559)$ & 0.051 & $4.86(0.991,23.8)$ \\
\hline \multicolumn{7}{|l|}{ Shoes wearing habit } \\
\hline Sometimes & $21(24.7)$ & $64(75.3)$ & $<0.001^{*}$ & $5.344(2.154,13.256)$ & $0.001^{* *}$ & $6.06(2.6,14.13)$ \\
\hline Usually & $27(17.3)$ & $129(82.7)$ & $0.006^{*}$ & $3.409(1.403,8.125)$ & & \\
\hline Regularly & $7(5.8)$ & $114(94.2)$ & & 1 & & 1 \\
\hline \multicolumn{7}{|l|}{ Proper latrine usage } \\
\hline No & $27(16.6)$ & $136(83.4)$ & 0.511 & $1.121(0.683,2.154)$ & & \\
\hline Yes & $28(14.1)$ & $171(85.9)$ & & 1 & & \\
\hline \multicolumn{7}{|c|}{ Swimming in river water } \\
\hline No & $13(8.7)$ & $136(91.3)$ & & 1 & & 1 \\
\hline Yes & $42(19.7)$ & $171(80.3)$ & 0.005 & $0.389(0.201,0.754)$ & 0.997 & \\
\hline \multicolumn{7}{|c|}{ Crossing a river with barefoot } \\
\hline No & $21(14.4)$ & $125(85.6)$ & & 1 & & \\
\hline Yes & $34(15.7)$ & $182(84.3)$ & 0.724 & $0.899(0.499,1.622)$ & & \\
\hline \multicolumn{7}{|c|}{ Drinking water source } \\
\hline Tap & $27(12.1)$ & $196(87.9)$ & & 1 & & 1 \\
\hline River/well & $28(20.1)$ & $111(79.9)$ & $0.040^{*}$ & $1.831(1.028,3.263)$ & 0.998 & \\
\hline
\end{tabular}

Note: ${ }^{\circledR}$ No $(\%),{ }^{*}$ statistically significant at $P<0$. 
methods, water velocity, lifestyles [26], local endemicity of the parasite [26], and temperature [28].

On the contrary, the schistosome prevalence of the present study (15.2\%) was higher than the prevalence in Egypt (6\%) [24]; the regional (36.4\%) and zonal (0.5 to 40.1\%) prevalences [12], and among Kindergarten Children in Mekelle Town (0.25\%) [29]. This variation might be due to variations in sociodemographic factors, immune status of participants, distance from the water source and frequency of contact with infected water, ecological distribution of intermediate host (snail), local endemicity of the parasite, sample size, and awareness regarding the transmission and prevention of S. mansoni infection [2].

The age groups 7 to 10 years were more infected with $S$. mansoni than the 11 to 14 and $\geq 15$-year-olds. This finding was in agreement with the findings of $[4,21,27]$. The reason why children aged from 7 to 10 years were more likely to have $S$. mansoni infection might be because children of these age groups are naturally active and have a strong affinity for water-related activities and the responsibility to look after cattle in wetland areas. On the other hand, the relatively low prevalence of $S$. mansoni infection among school children in the age group between 11 to 14 and $\geq 15$ might be due to praziquantel drug mass deworming activities for school children [30]. However, the age-specific result of the present study was in contrast to the findings reported by researchers [20,31].

The other significant factor associated with S. mansoni infection in this study was the grade level of the study participants. Students with grade levels of 1 to 4 were more likely to be exposed to $S$. mansoni infection than grade levels 5 to 8 . The possible reason may be a lower level of awareness about personal hygiene and the mode of $S$. mansoni transmission among children whose grade levels were 1 to 4 . This finding was in agreement with the findings of $[4,10]$. But it disagreed with the findings of $[20,26]$.

In the school-specific prevalence of $S$. mansoni infection, the highest prevalence was observed in Chifarit primary school (30.3\%), followed by Tikur Wuha (17.1\%), Jiga 01 primary school (10.6\%). This variation could be due to continuous water contact habits and barefoot irrigation activities. All participants in Chifarit School came from farmer families. In their village and near to their school, there is irrigation canal water. They used this water for different purposes like cloth and body washing, cleaning utensils, to grow vegetables, and to clean their classrooms. They cross the water when they come to school and go to their homes. There is also a wetland near their school and villages.

The prevalence of $S$. mansoni infection among female and male participants was $15.4 \%$ and $15.1 \%$, respectively, but no statistical difference was observed. This agrees with the findings of $[4,26]$ but disagrees with the findings of $[2,10,21]$.

In this study, students who used to wash their clothes in a river or irrigation canal water and had a habit of taking bath in a river were more likely to acquire $S$. mansoni infection and the difference was statistically significant $(P=0.008)$. This is supported by the study conducted by $[10,21,26$, 27]. Swimming, washing clothes in rivers, and bathing in river waters were reported to be significantly associated with a high risk of S. mansoni infection [21]. Likewise, the findings in the present study showed that students who had the habit of swimming in rivers and irrigation canals were more likely to acquire $S$. mansoni infection and the difference was statistically significant $(P=0.005)$. This coincides with reports by $[4,21,26,27,31]$.

In the present study, there was variation in $S$. mansoni infection related to the habit of shoes wearing among the participant school children. Participants who wore shoes sometimes were 5.34 times more likely for $S$. mansoni infection than participants who wore shoes regularly. This variation was statistically significant $(P=0.001)$ and agrees with research findings reported from School Children in Sanja Town [26] and School Children in Mana District [31]. Besides, participants from Chifarit primary school wore shoes sometimes since there is a wetland in their village. Especially, during the summer season, the wetland was not suitable to wear shoes. This could increase the probability of school children to be infected with S. mansoni. Similarly, the habit of shoes wearing among school children was one of the most risk factors for the prevalence of $S$. mansoni in the study area.

\section{Conclusion}

The overall prevalence of $S$. mansoni infection among students from the three schools was medium (15.2\%). The highest prevalence was registered among Chifarit primary school students $(30.3 \%)$ followed by Tikur Wuha primary school $(17.1 \%)$ and Jiga 01 primary school (10.6\%) in descending order. Grades 1-4, ages 7 to 10 and 11 to 14 , Chifarit primary school, contact with river water, and the irregular wearing of shoes were risk factors for $S$. mansoni infection.

5.1. Limitation of the Study. Not using Kato-Katz slides as a golden standard may underestimate the results.

\section{Abbreviations}

AOR: Adjusted odds ratio

BDU: Bahir Dar University

CDC: Center for Disease Control

CI: Confidence interval

COR: Crude odds ratio

CSA: Central Statistics Agency of Ethiopia

NTDs: Neglected tropical diseases

Spp: Species

SPSS: Statistical package for social science

WHO: World Health Organization.

\section{Data Availability}

All datasets generated and analyzed during the study are presented in the paper.

\section{Ethical Approval}

Ethical clearance was obtained from the College of Sciences research committee of Bahir Dar University, and permission 
letter was also obtained from the West Gojjam zone Health office and education authorities before conducting the study. Written informed consent was obtained from the parents/guardians of the children. Those children who were positive for schistosomiasis were linked to the Jiga health center to be treated. Data were confidential and used only for the purpose of this study.

\section{Conflicts of Interest}

The authors declare that they have no competing interests.

\section{Authors' Contributions}

KW participated in the design of the study, performed the experiments, analyzed the data, and made inputs in the manuscript write-up. DD supervised, reviewed, and provided inputs to the manuscript. Both authors read and approved the final manuscript.

\section{Acknowledgments}

The authors are grateful to all the school children who gave their consent to participate in the study. Our special thanks to the Chief of Centre, nurses, and laboratory technicians of the Jiga health center for their cooperation and contribution. We also thank school directors and teachers of the three schools for their contributions during sociodemographic data collection. This work was supported by the Amhara education office and Bahir Dar University.

\section{References}

[1] WHO, "Neglected tropical diseases," 2020, June 2020, https:// www.who.int/neglected_diseases/diseases/en/.

[2] M. Dufera, B. Petros, B. Erko, N. Berhe, and S. G. Gundersen, "Schistosoma mansoni infection in Finchaa sugar estate: public health problem assessment based on clinical records and parasitological surveys, western Ethiopia," Science, Technology, and Arts Research Journal, vol. 3, no. 2, pp. 155-161, 2014.

[3] WHO, "Schistosomiasis," 2020, June 2020, https://www.who. int/news-room/fact-sheets/detail/schistosomiasis.

[4] W. Awoke, M. Bedimo, and M. Tarekegn, "Prevalence of Schistosomiasis and Associated Factors among Students Attending Elementary Schools in Amibera District, Ethiopia," Open Journal of Preventive Medicine, vol. 3, no. 2, pp. 199-204, 2013.

[5] N. Negussu, B. Mengistu, B. Kebede et al., "Ethiopia schistosomiasis and soil-transmitted helminths control program: progress and prospects," Ethiopian Medical Journal, vol. 55, Supplement 1, p. 75, 2017.

[6] B. Chala and W. Torben, "An epidemiological trend of urogenital schistosomiasis in Ethiopia," Frontiers in Public Health, vol. 6, p. 60, 2018.

[7] Global Atlas of Helminth Infections, "The Global Burden of Disease study estimates the magnitude of health loss due to diseases and injuries," 2020, June 2020, http://www.thiswor myworld.org/worms/global-burden.

[8] M. T. Inobaya, R. M. Olveda, T. N. Chau, D. U. Olveda, and A. G. Ross, "Prevention and control of schistosomiasis: a cur- rent perspective," Research and Reports in Tropical Medicine, vol. 2014, no. 5, p. 65, 2014.

[9] R. Sturrock, "Schistosomiasis epidemiology and control: how did we get here and where should we go?," Memórias do Instituto Oswaldo Cruz, vol. 96, suppl, pp. 17-27, 2001.

[10] T. Essa, Y. Birhane, M. Endris, A. Moges, and F. Moges, "Current status of Schistosoma mansoni infections and associated risk factors among students in Gorgora town, Northwest Ethiopia," International Scholarly Research Notices, vol. 2013, Article ID 636103, 7 pages, 2013.

[11] H. Sady, H. M. Al-Mekhlafi, R. Ngui et al., "Detection of Schistosoma mansoni and Schistosoma haematobium by real-time PCR with high resolution melting analysis," International Journal of Molecular Sciences, vol. 16, no. 7, pp. 1608516103, 2015.

[12] A. W. Nute, T. Endeshaw, A. E. Stewart et al., "Prevalence of soil-transmitted helminths and Schistosoma mansoni among a population-based sample of school-age children in Amhara region, Ethiopia," Parasites \& Vectors, vol. 11, no. 1, p. 431, 2018.

[13] F. Shewakena, H. Kloos, F. Abebe, and H. Birrie, "Schistosoma mansoni infection in Jiga town, Gojam Administrative Region," The Ethiopian Journal of Health Development, vol. 9, no. 1, 1995.

[14] Central Statistical Agency (CSA), "Population and Housing Census 2007 Report, Amhara, Part I: population Size and Characteristics," 2010, June 2020, https://catalog.ihsn.org/ index.php/catalog/3583/download/50095.

[15] WikiHow, "How to calculate sample size," 2019, June 2020, https://www.wikihow.com/Calculate-Sample-Size.

[16] "Sample size calculator," 2020, June 2020, https://www.calcula tor.net/sample-size-calculator.html.

[17] WHO Expert Committee, "Prevention and control of schistosomiasis and soil-transmitted helminthiasis," World Health Organization Technical Report Series, vol. 912, 2002.

[18] World Health Organization, Basic Laboratory Methods in Medical Parasitology, World Health Organization, 1991.

[19] S. A. Ismail, W. Kamal, and H. K. Salem, "Schistosoma prevalence world-wide," 2016, http://www.smgebooks.com.

[20] H. Desta, G. Bugssa, and B. Demtsu, "The current status of Schistosoma mansoni infection among school children around Hizaty Wedicheber Microdam in Merebmieti, Ethiopia," Journal of Bacteriology \& Parasitology, vol. 5, no. 5, p. 1, 2014.

[21] E. Molla, Prevalence of Schistosomiasis and Associated Risk Factors among Students in Addis Zemen Town, North West Ethiopia, [M.Sc. thesis], Bahir Dar University, Ethiopia, 2017.

[22] D.-D. Lin, J.-X. Liu, Y.-M. Liu et al., The Control of Schistosomiasis: Report of a WHO Expert Committee, WHO technical Rep. Series 728, Geneva, 1985.

[23] B. Mengitsu, O. Shafi, B. Kebede et al., "Ethiopia and its steps to mobilize resources to achieve 2020 elimination and control goals for neglected tropical diseases: spider webs joined can tie a lion," International Health, vol. 8, Supplement 1, pp. i34-i52, 2016.

[24] M. L. Steinauer, M. S. Blouin, and C. D. Criscione, "Applying evolutionary genetics to schistosome epidemiology," Infection, Genetics and Evolution, vol. 10, no. 4, pp. 433-443, 2010.

[25] M. Bajiro, D. Dana, M. Ayana et al., "Prevalence of Schistosoma mansoni infection and the therapeutic efficacy of praziquantel among school children in Manna District, Jimma Zone, southwest Ethiopia," Parasites \& Vectors, vol. 9, no. 1, p. $560,2016$. 
[26] L. Worku, D. Damte, M. Endris, H. Tesfa, and M. Aemero, "Schistosoma mansoni infection and associated determinant factors among school children in Sanja Town, Northwest Ethiopia," Journal of Parasitology Research, vol. 2014, Article ID 792536, 7 pages, 2014.

[27] G. Alebie, B. Erko, M. Aemero, and B. Petros, "Epidemiological study on Schistosoma mansoni infection in Sanja area, Amhara region, Ethiopia," Parasites \& Vectors, vol. 7, no. 1, p. 15, 2014.

[28] L. Workineh, M. Yimer, W. Gelaye, and D. Muleta, "The magnitude of Schistosoma mansoni and its associated risk factors among Sebatamit primary school children, rural Bahir Dar, Northwest Ethiopia: a cross-sectional study," BMC Research Notes, vol. 12, no. 1, p. 447, 2019.

[29] M. Alemu, H. Bedemo, G. Bugssa, S. Bayissa, and K. Tedla, "Epidemiology of intestinal parasite infections among kindergarten children in Mekelle Town, Northern Ethiopia," International Journal of Pharmaceutical Sciences and Research, vol. 6, no. 11, pp. 1392-1396, 2015.

[30] L. Legesse, B. Erko, and A. Hailu, "Current status of intestinal Schistosomiasis and soiltransmitted helminthiasis among primary school children in Adwa Town, Northern Ethiopia," Ethiopian Journal of Health Development, vol. 24, no. 3, 2011.

[31] M. Bajiro and S. Tesfaye, "Schistosoma mansoni infection prevalence and associated determinant factors among school children in Mana District, Jimma Zone, Oromia Region, South West Ethiopia," Journal of Bacteriology \& Parasitology, vol. 8, no. 329 , p. 2, 2017. 